\title{
An Axiomatic Characterization of the Leximin Choice Rule
}

\author{
Osamu Mori \\ Aomori Public University, Aomori, Japan \\ Email: morsam-31@b.nebuta.ac.jp
}

How to cite this paper: Mori, O. (2019) An Axiomatic Characterization of the Leximin Choice Rule. Theoretical Economics Letters, 9, 2165-2175.

https://doi.org/10.4236/tel.2019.96137

Received: July 19, 2019

Accepted: August 27, 2019

Published: August 30, 2019

Copyright $\odot 2019$ by author(s) and Scientific Research Publishing Inc. This work is licensed under the Creative Commons Attribution International License (CC BY 4.0). http://creativecommons.org/licenses/by/4.0/

\begin{abstract}
This study discusses the characterization of the lexicographic maximin (leximin) choice rule using an axiom related to the Lorenz criterion, which states that a utility vector that Lorentz dominates a solution vector should not be Pareto dominated by any feasible vector, named Pareto Undominatedness of Lorenz-superior Distribution (PULSD). PULSD in itself does not imply that a solution vector of the choice rule is Lorenz undominated by any utility vectors in the feasible set. Therefore, we may say that the PULSD's requirement for inequality aversion is weak. Indeed, PULSD is consistent with the utilitarian choice rule, which is viewed as indifferent to equity. We show that the leximin choice rule is characterized by PULSD, combined with the Suppes-Sen optimality and the common ordinal invariance axiom.
\end{abstract}

\section{Keywords}

The Leximin Choice Rule, The Lorenz Criterion, Suppes-Sen Optimality, The Common Ordinal Invariance Axiom

\section{Introduction}

We consider a choice problem to select the optimal distribution from a set of feasible alternatives that represent utility vectors. The social planner may establish a choice rule, taking into account the equity property as well as the efficiency property. For the efficiency notion, the Suppes-Sen optimality (SSO) is well known and standard ${ }^{1}$. SSO requires that a solution utility vector be not Suppes-Sen dominated by any utility vector $s$ in the feasible set ${ }^{2}$. SSO is regarded as representing the property of impartiality in the use of the strong Pareto opti- 
mality ${ }^{3}$. For the equity notion, the Lorentz criterion is renowned ${ }^{4}$. In general, for two utility vectors $x$ and $y, x$ is regarded as more inequality averse or equitable than $y$ if $x$ Lorenz dominates $y$. A solution vector may be required to be Lorenz undominated by any utility vectors in the feasible set, which we refer to as Lorenz undominatedness (LU). In this sense, $\mathrm{LU}$ requires that any vector that is more inequality-averse or equitable than a solution vector is not included in the feasible set.

Ok [11] proposed the notion that is obtained by combining LU with Pareto optimality, which is referred to as Lorenz-Pareto optimality (LPO). LPO requires that a solution be neither Pareto dominated nor Lorenz dominated by any vector in the feasible set. LPO is a refined notion of Pareto optimality containing the inequality-averse property. As Ok [11] demonstrated, provided that $S$ is a compact and comprehensive set, GLO implies and is implied by the generalized Lorenz optimality (GLO), which requires that a solution vector be not generalized Lorenz dominated by any feasible vector ${ }^{5}$.

The utilitarian choice rule, which aims to maximize the total utility and is viewed as indifferent to equality or equity, is inconsistent with LU, LPO, and GLO. In contrast, the lexicographic maximin (leximin) choice rule, which is regarded as the representative egalitarian rule, satisfies all of them ${ }^{6}$. However, the leximin choice rule may be characterized without directly requiring a solution vector to be Lorenz undominated by any vector in the feasible set. In this study, we focus on the characterization of the leximin choice rule. Instead of LU, LPO, or GLO, we use the axiom, what we refer to as Pareto undominatedness of Lorenz-superior distribution (PULSD), which requires that a feasible or infeasible utility vector that Lorentz dominates a solution vector be Pareto undominated by any vector in the feasible set. Note that PULSD is consistent with the utilitarian choice rule.

Then, we introduce an invariance axiom, Common Ordinal Invariance (COI), which is based on the weak ordinal invariance axiom that Thomson [18] used to characterize the lexicographic egalitarian bargaining solution ${ }^{7}$. COI states that under any positive transformation of the feasible utilities the choice solution utility vectors should be transformed accordingly. COI is regarded as the requirement for consistency with respect to any positive transformation.

Herein, the leximin choice rule is characterized by combining SSO, PULSD, and $\mathrm{COI}^{8}$.

${ }^{3}$ Mariotti [8].

${ }^{4}$ See Dasgupta, Sen, and Starrett [3] and Sen [14]. The Lorenz criterion they used is based on the well-known notion of Lorenz curve representing inequality with respect to income distribution. However, we use the Lorenz criterion with respect to utility distribution throughout the paper. See also Ok[11]

${ }^{5}$ See proposition 1 of Ok [11] (p. 309). For the notion of the generalized Lorenz dominance, see Shorrocks [15], Madden [6], and Ok [11].

${ }^{6}$ It will be shown later.

${ }^{7}$ See also Nielsen [10].

${ }^{8}$ In Mori [9], the leximin bargaining solution is characterized in the case of the two-person bargaining problem. The present study shows that the leximin choice rule can be characterized using similar axioms within the framework of a choice problem of society comprising $n(\geq 3)$ individuals. 
The remainder of the paper is organized as follows. Section 2 presents the preliminaries of the analysis and the axioms. Section 3 provides a theorem and two corollaries about the characterization of the leximin choice rule. Section 4 provides concluding remarks.

\section{Preliminaries and Axioms}

We consider a society comprising $n(\geq 3)$ individuals. A choice problem is defined as a set of feasible utilities $S \subset \mathbb{R}^{n} . S$ is assumed to be closed and bounded above. Let $\mathbb{C}^{n}$ be the class of choice problems satisfying this condition. A choice rule is a nonempty correspondence $F: \mathbb{C}^{n} \rightarrow \mathbb{R}^{n}$ such that for every $S \in \mathbb{C}^{n}, F(S) \subset S$. We refer to $t \in F(S)$ as a choice solution utility vector or, in short, a solution vector.

The following vector notations are used throughout. For $s, r \in \mathbb{R}^{n}, s \geq r$ if $s_{i} \geq r_{i}$ for each $i, s>r$ if $s_{i} \geq r_{i}$ for all $i$ and $s \neq r$, and $s \gg r$ if $s_{i}>r_{i}$ for each $i$. For each $p \in\{1, \cdots, n !\}$, a function $\pi^{p}: \mathbb{R}^{n} \rightarrow \mathbb{R}^{n}$, is defined such that $\pi^{p}(s)=\left(\pi_{1}^{p}(s), \cdots, \pi_{n}^{p}(s)\right)$ where $\pi_{i}^{p}(s)=s_{\sigma^{p}(i)}$ for a permutation $\sigma^{p}:\{1, \cdots, n\} \rightarrow\{1, \cdots, n\}$. Let $\pi^{1}(S)$ be such that $\sigma^{1}$ is the identity permutation, i.e., $\pi^{1}(s)=s$, and $s_{1}^{\uparrow}, \cdots, s_{n}^{\uparrow}$ be the elements of $s$ listed in ascending order where $s_{i}^{\uparrow}$ denotes the $i$-th element from the minimum one, i.e., $s_{1}^{\uparrow} \leq \cdots \leq s_{1}^{\uparrow}$.

The lexicographic maximin (leximin) rule is the correspondence $L_{\min }: \mathbb{C}^{n} \rightarrow \mathbb{R}^{n}$, which is defined as follows ${ }^{10}$ :

$$
\begin{aligned}
L_{\min }(S)= & \left\{e \in S \mid s \notin S \text { if } e_{1}^{\uparrow}<s_{1}^{\uparrow}, e_{1}^{\uparrow}=s_{1}^{\uparrow} \text { and } e_{2}^{\uparrow}<s_{2}^{\uparrow}, \cdots,\right. \\
& \text { or } \left.e_{1}^{\uparrow}=s_{1}^{\uparrow}, \cdots, e_{n-1}^{\uparrow}=s_{n-1}^{\uparrow} \text {, and } e_{n}^{\uparrow}<s_{n}^{\uparrow}\right\} .
\end{aligned}
$$

Note that $L_{\min }(S) \neq \varnothing$ since $S$ is closed and bounded above.

The impartiality and efficiency property that is generally imposed on a solution $F$ is as follows:

Suppes-Sen Optimality (SSO): For any $t \in F(S)$ and for any $s \in \mathbb{R}^{n}$, if $s>\pi^{p}(t)$ for some $p \in\{1, \cdots, n !\}$, then $s \notin S^{11}$.

Following Madden [6] and Mariotti [8], we say that $s$ Suppes-Sen dominates $t$ if $s>\pi^{p}(t)$ for some $p \in\{1, \cdots, n !\}^{12}$. SSO requires that there be no utility vector $s$ in the feasible set that Suppes-Sen dominates any solution utility vector $t$.

A standard notion used for representing the inequality aversion property is Lorenz dominance (LD), which is defined as follows: A utility vector $r \in \mathbb{R}^{n}$ Lorenz dominates $s \in \mathbb{R}^{n}$ if and only if $\sum_{i=1}^{n} s_{i}=\sum_{i=1}^{n} r_{i}$, and for all $k \leq n$, $\sum_{i=1}^{k} s_{i} \leq \sum_{i=1}^{k} r_{i} \quad$ with at least one $k<n$ such that $\sum_{i=1}^{k} s_{i}<\sum_{i=1}^{k} r_{i}{ }^{13}$. In words, LD

${ }^{9}$ The notation of $s_{i}^{\uparrow}$ follows Mariotti [7].

${ }^{10}$ Sen [13].

${ }^{11}$ Madden [6] p.250.

${ }^{12}$ As is well-known, the notion of Suppes-Sen dominance is based on Suppes [16] and Sen [13].

${ }^{13}$ Dasgupta, Sen, and Starrett [3], Sen [14]. Note that they state Lorenz dominance in terms of income, but we define it in terms of utility. See also Ok [11]. 
says that between two utility vectors whose total utilities are equal, for all $k$ lower utility individuals of the population $n, r$ does not yield a smaller sum of utilities than $s$, and for some $k$ lower utility individuals of the population $n, r$ yields a larger sum of utilities than $s$. We can say that $r$ is more inequality averse or equitable than $s$. From Hardy, Littlewood, and Polya's [5] theorem, LD is equivalent to the condition that a bistochastic matrix $Q$ exists such that $r=Q s$ and $r$ is not equal to a permutation of $s^{14}$. From the theorem of Birkhoff-von Neumann, $Q s$ represents a convex combination of permutations of $s^{15}$. Therefore, the set of vectors that Lorenz dominate $s, L(s)$, can be expressed as follows ${ }^{16}$ :

$$
\begin{gathered}
L(s)=\left\{r \in \mathbb{R}^{n} \mid r=\sum_{p=1}^{n !} \lambda_{p} \pi^{p}(s) \text { for } \lambda=\left(\lambda_{1}, \cdots, \lambda_{n !}\right) \in \Lambda\right. \\
\text { and } \left.r \neq \pi^{p}(s), p=1, \cdots, n !\right\}
\end{gathered}
$$

where $\Lambda=\left\{\lambda \mid 0 \leq \lambda_{p}<1, p=1, \cdots, n !, \sum_{p=1}^{n !} \lambda_{p}=1\right\}$

Note that for $r \in L(s)$ each $r_{i}, i=1, \cdots, n$ is a weighted average of $s_{1}, \cdots, s_{n}$ We call $r \in L(s)$ a Lorenz-superior distribution with respect to $s \in \mathbb{R}^{n}$.

An axiom imposing the inequality aversion property on a solution vector is as follows.

Lorenz Undominatedness (LU): For any $t \in F(S), L(t) \cap S=\varnothing$.

LU requires that any utility vector $s \in L(t)$ for a solution utility vector $t$ be not contained in the feasible set $S$. This implies that the distributional disparity of a solution utility vector should be reduced to the minimum ${ }^{17}$.

The notion of LD concerns the relation between two distributions whose total utilities are equal. When comparing two utility vectors whose total utilities are not equal, we can use the notion of the generalized Lorenz dominance (GLD), which is defined as follows ${ }^{18}$ : A utility vector $r \in \mathbb{R}^{n}$ generalized Lorenz dominates $s \in \mathbb{R}^{n}$ if and only if for all $k \leq n, \sum_{i=1}^{k} s_{i} \leq \sum_{i=1}^{k} r_{i}$ with at least one $k \leq n$ such that $\sum_{i=1}^{k} s_{i}<\sum_{i=1}^{k} r_{i}$. From the equivalence theorem on LD mentioned above, the following alternative expression is possible: A utility vector $r \in \mathbb{R}^{n}$

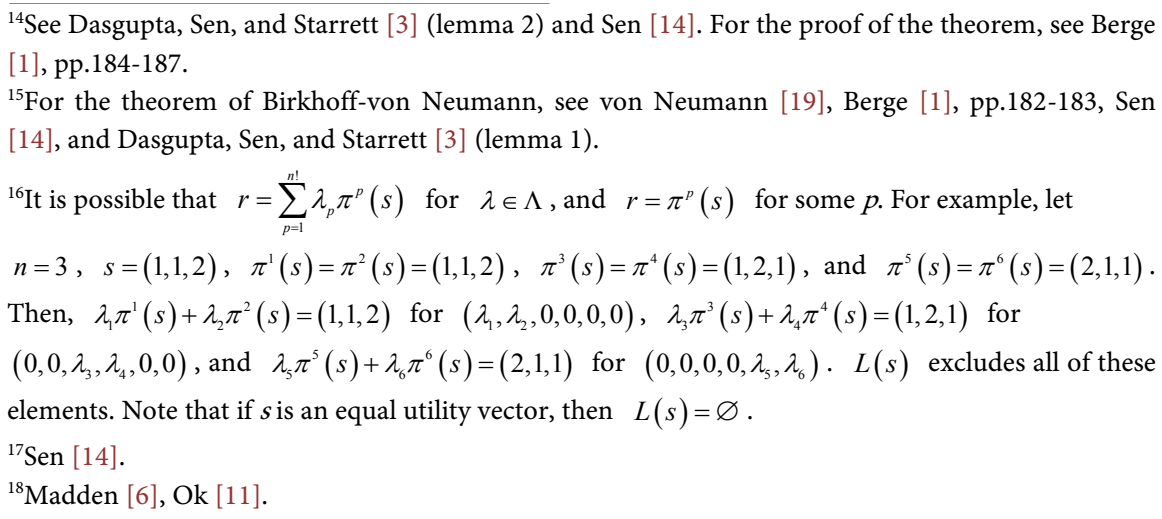


generalized Lorenz dominates $s \in \mathbb{R}^{n}$ if and only if there is a bistochastic matrix $Q$ such that $r \geq Q s$ with $r>Q s$ if $Q s$ is equal to a permutation of $s^{19}$.

It is legitimate to require that a solution vector be not generalized Lorenz dominated by any feasible vectors. We refer to this requirement as the generalized Lorenz optimality (GLO) ${ }^{20}$.

Generalized Lorenz Optimality (GLO): For any $t \in F(S)$, for some $r \in L(t)$, and for any $s \in \mathbb{R}^{n}$, if $s \geq r$, or $s>\pi^{p}(t)$ for $p \in\{1, \cdots, n !\}$, then $s \notin S$.

Ok [11] discussed the notion of the Lorenz-Pareto frontier, which is a set of Pareto optimal vectors that are not Lorenz dominated by any utility vectors in the feasible set, and introduced the notion of Lorenz-Pareto optimality, which is defined in our framework as follows.

Lorenz-Pareto Optimality (LPO): For any $t \in F(S)$ and for any $s \in \mathbb{R}^{n}$, if $s>t$ or $s \in L(t)$, then $s \notin S$.

Ok [11] argued that for two Pareto optimal vectors $x$ and $y$, and a vector $z$ in the feasible set, when $x$ Pareto dominates $z$ which in turn Lorenz dominates $y$, it can be said that $x$ clearly dominates $y^{21}$. Ok [11] states that if this is the case, it may be said that a Lorenz-Pareto improvement over $y$ is feasible, and that a vector $u$ is Lorenz-Pareto optimal if and only if a Lorenz-Pareto improvement over $u$ is infeasible $\mathrm{e}^{22}$. Based on the discussion, we say that it is natural to consider that a feasible or infeasible distribution $r \in \mathbb{R}^{n}$ that (strictly) Lorenz dominates a solution vector $t$, should be Pareto undominated by any distribution in the feasible set. In other words, there should be no feasible distribution that (strictly) Pareto dominates a Lorenz-superior distribution with respect to a solution vector. We introduce the following axiom, which we refer to as the Pareto undominatedness of Lorenz-superior distribution.

Pareto Undominatedness of Lorenz-Superior Distribution (PULSD): For any $t \in F(S)$, for any $r \in L(t)$, and for any $s \in \mathbb{R}^{n}$, if $s>r$, then $s \notin S^{23}$.

Satisfying PULSD means that no utility vector is more efficient than any utility vector that is more equitable than a solution vector. Note that PULSD does not require that $r \in L(t)$ for a solution utility vector $t$ be excluded from $S$. In this sense, PULSD's requirement for equity is relatively weak.

It is certain that GLO implies SSO, LU, LPO and PULSD. Indeed, GLO is equivalent to the combination of SSO, LU, and PULSD.

Moreover, we introduce the following invariance axiom.

Common Ordinal Invariance (COI): Let $\tau: \mathbb{R} \rightarrow \mathbb{R}$ be a strictly increasing function and let $S^{\prime}=\left\{\left(\tau\left(s_{1}\right), \cdots, \tau\left(s_{n}\right)\right) \mid s \in S\right\}$. For $S, S^{\prime} \in \mathbb{C}^{n}$, if $t \in F(S)$, ${ }^{19}$ Madden [6] p.250.

${ }^{20}$ Madden [6] refers to a feasible utility vector which is not generalized Lorenz dominated by any other feasible vector as a generalized Lorenz optimum (p.251).

${ }^{21}$ Ok [11] pp.307-308.

${ }^{22}$ It seems that, strictly speaking, what Ok [11] calls the feasibility of a Lorenz-Pareto improvement over $y$ means that there is a vector $x$ such that $x$ weakly Pareto dominates $z$ which strictly Lorenz dominates $y$, or, $x$ strictly Pareto dominates $z$ which weaklyLorenz dominates $y$.

${ }^{23}$ PULSD can be also defined as the following condition: For any $t \in F(S)$, for $s \in \mathbb{R}^{n}$ such that $s>t$, if $r \in L(s)$, then $r \notin S$. 
then $t^{\prime}=\left(\tau\left(t_{1}\right), \cdots, \tau\left(t_{n}\right)\right) \in F\left(S^{\prime}\right)^{24}$.

COI requires that under any positive monotonic transformation of the feasible utilities the choice solution utility vectors be transformed accordingly. Such transformations preserve the order of utility levels regarding the intrapersonal and interpersonal comparisons of utilities since the positive monotonic transformation of vectors implies that for any $r, s \in \mathbb{R}^{n}$ (maybe $r=s$ ), for $i, j \in\{1, \cdots, n\}, \quad r_{i}<s_{j}$ if and only if $\tau\left(r_{i}\right)<\tau\left(s_{j}\right)$ and $r_{i}=s_{j}$ if and only if $\tau\left(r_{i}\right)=\tau\left(s_{j}\right)$. This axiom is usually said to correspond to the supposition that individual utilities are ordinally measurable and interpersonally full-comparable. However, it should be noted that COI is consistent with the supposition that individual utilities are cardinally measurable and interpersonally full-comparable. In fact, COI is satisfied whenever for all $i, \tau\left(s_{i}\right)=\alpha+\beta s_{i}, \alpha, \beta \in \mathbb{R}, \beta>0{ }^{25}$. Even in such a case, social planner may disregard how large or small the intrapersonal and interpersonal utility differences are. Hence, COI should be considered as the consistency condition with respect to any positive transformation.

\section{Characterizing the Leximin Choice Rule}

It is well-known and easily demonstrated that $L_{\min }(S)$ satisfies GLO.

Lemma: $L_{\min }(S)$ implies GLO.

Proof. Suppose by contradiction that there exists $s \in S$, which generalized Lorenz dominates $e \in L_{\min }(S)$. It must be that $\sum_{i=1}^{j} e_{i}^{\uparrow} \leq \sum_{i=1}^{j} s_{i}^{\uparrow}$ for all $j \leq n$ and $\sum_{i=1}^{j} e_{i}^{\uparrow}<\sum_{i=1}^{j} s_{i}^{\uparrow}$ for some $j \leq n$. Let $h=\min \left\{j \in\{1, \cdots, n\} \mid \sum_{i=1}^{j} e_{i}^{\uparrow}<\sum_{i=1}^{j} s_{i}^{\uparrow}\right\}$. It follows that $e_{i}^{\uparrow}=s_{i}^{\uparrow}$ for all $i<h$ and $e_{h}^{\uparrow}<s_{h}^{\uparrow}$. That is a contradiction since $e \in L_{\text {min }}(S)$. Hence, $L_{\text {min }}(S)$ implies GLO. Q.E.D.

However, LU combined with SSO and COI is insufficient for $L_{\min }(S)$ to be the unique rule for the choice problem. To illustrate this, we define $\hat{S} \subset \mathbb{R}^{n}$ as a set composed of two vectors $a, b \in \mathbb{R}^{n}$ such that $b_{1}^{\uparrow}>a_{1}^{\uparrow}$, $b_{2}^{\uparrow}=\cdots=b_{n-1}^{\uparrow}<a_{2}^{\uparrow}=\cdots=a_{n-1}^{\uparrow}$, and $b_{n}^{\uparrow}>a_{n}^{\uparrow}$. Then, we consider the following choice rule:

$$
\hat{F}(S)= \begin{cases}\arg \max _{x \in S} x_{2}^{\uparrow} & \text { if } S=\hat{S} \\ L_{\min }(S) & \text { if } S \neq \hat{S}\end{cases}
$$

Since $a \notin L(b), b \notin L(a)$ for $a, b \in \hat{S}, \hat{F}(S)$ satisfies LU. Also, it is easily checked that $\hat{F}(S)$ satisfies $\mathrm{SSO}$ and $\mathrm{COI}^{26}$.

\footnotetext{
${ }^{24} \mathrm{As}$ mentioned in Introduction, it follows the weak ordinal invariance axiom in Thomson (p.1256) [18].

${ }^{25}$ For the discussion on the invariance axioms, see Bossert and Weymark [2].

${ }^{26}$ Note that $\hat{F}(S)$ violates the independence of irrelevant alternatives axiom (IIA), which requires that for any $S, S^{\prime} \in \mathbb{C}^{n}$, if $S^{\prime} \subset S$ and $F(S) \in S^{\prime}$, then $F\left(S^{\prime}\right)=F(S)$. (For IIA see Thomson [17].) For letting $\hat{S}=\{a, b\}$ and $S=\{a, b, c\}$ where $b_{1}^{\uparrow}>a_{1}^{\uparrow}>c_{1}^{\uparrow}, b_{2}^{\uparrow}=\cdots=b_{n-1}^{\uparrow}<a_{2}^{\uparrow}=\cdots=a_{n-1}^{\uparrow}$, and $b_{n}^{\uparrow}>a_{n}^{\uparrow}$, it follows that $\hat{F}(\hat{S})=a$, but $\hat{F}(S)=b$.
} 
We characterize $L_{\min }(S)$ using PULSD.

Theorem: $A$ choice rule $F(S)$ on $\mathbb{C}^{n}$ satisfies SSO, PULSD, and COI if and only if it is the leximin choice rule $L_{\min }(S)$.

Proof. First, we demonstrate that $L_{\text {min }}(S) \subset F(S)$. By Lemma $L_{\text {min }}(S)$ satisfies GLO, which implies SSO and PULSD. Since any positive transformation of utilities preserves the order of utility levels, it follows that $e \in L_{\min }(S)$ if and only if $e^{\prime} \in L_{\min }\left(S^{\prime}\right)$ where $e^{\prime}=\left(\tau\left(e_{1}\right), \cdots, \tau\left(e_{n}\right)\right)$, $S^{\prime}=\left\{\left(\tau\left(s_{1}\right), \cdots, \tau\left(s_{n}\right)\right) \mid s \in S\right\}$, and $\tau$ is a strictly increasing function. Therefore, $L_{\text {min }}(S)$ satisfies COI.

Second, we prove that $F(S) \subset L_{\text {min }}(S)$. Let a choice rule $F(S)$ satisfy all axioms in the theorem. We suppose by contradiction that $F(S) \not \subset L_{\min }(S)$. Consider $t$ such that $t \in F(S)$ and $t \notin L_{\min }(S)$, and $e \in L_{\min }(S)$. Note that $t$ is not a permutation of $e$. Our strategy for the proof is to demonstrate that a positive monotonic transformation of $t, t^{\prime}=\left(\tau\left(t_{1}\right), \cdots, \tau\left(t_{n}\right)\right)$, satisfying COI violates PULSD. Let $k=\min \left\{i \in\{1, \cdots, n\} \mid t_{i}^{\uparrow}<e_{i}^{\uparrow}\right\}$. There exists $k$ since $e$ is not a permutation of $t$ and $e$ satisfies SSO. It follows that $t_{i}^{\uparrow}=e_{i}^{\uparrow}$ for $i<k$ if $k \geq 2$. By SSO, there must exist $i \in\{k+1, \cdots, n\}$ such that $e_{i}^{\uparrow}<t_{i}^{\uparrow}$. Let $m=\min \left\{i \in\{k+1, \cdots, n\} \mid e_{i}^{\uparrow}<t_{i}^{\uparrow}\right\}$.
We define $\tau: \mathbb{R} \rightarrow \mathbb{R}$ as follows:

$$
\tau(x)= \begin{cases}x & \text { if } x<e_{k}^{\uparrow} \\ \varepsilon x+(1-\varepsilon) e_{k}^{\uparrow} & \text { if } e_{k}^{\uparrow} \leq x\end{cases}
$$

where $0<\varepsilon<\frac{1}{2^{n-m+1}-1} \frac{e_{k}^{\uparrow}-t_{k}^{\uparrow}}{t_{n}^{\uparrow}-e_{k}^{\uparrow}}$

We consider $S^{\prime}=\left\{\left(\tau\left(s_{1}\right), \cdots, \tau\left(s_{n}\right)\right)\right\}$ for $s \in S$. Letting $t^{\prime}=\left(t_{1}^{\prime}, \cdots, t_{n}^{\prime}\right)=\left(\tau\left(t_{1}\right), \cdots, \tau\left(t_{n}\right)\right), \quad e^{\prime}=\left(e_{1}^{\prime}, \cdots, e_{n}^{\prime}\right)=\left(\tau\left(e_{1}\right), \cdots, \tau\left(e_{n}\right)\right)$, by COI it follows that $t^{\prime} \in F\left(S^{\prime}\right), t^{\prime} \notin L_{\min }\left(S^{\prime}\right)$, and $e^{\prime} \in L_{\min }\left(S^{\prime}\right)$. Let $v=\left(v_{1}, \cdots, v_{n}\right)=\left(\tau\left(t_{1}^{\uparrow}\right), \cdots, \tau\left(t_{n}^{\uparrow}\right)\right)$, and $z=\left(z_{1}, \cdots, z_{n}\right)=\left(\tau\left(e_{1}^{\uparrow}\right), \cdots, \tau\left(e_{n}^{\uparrow}\right)\right)$. By the definition of $\tau$, the elements of $v$ and $z$ are in ascending order, i.e., $v_{1} \leq \cdots \leq v_{n}$ and $z_{1} \leq \cdots \leq z_{n}$, respectively. Note that $v_{i}=z_{i}$ for $i<k$, $v_{k}<z_{k}, \quad v_{i} \leq z_{i}$ for $i$ such that $k<i<m$, and $v_{m}>z_{m}$.

It should be noted that $L(v)=L\left(t^{\prime}\right)$, and $\pi^{p}(w) \in L(v)$ for any $w \in L(v)$ and for any $p \in\{1, \cdots, n !\}$. Therefore, $z>w$ implies that $e^{\prime}<\pi^{p}(w) \in L\left(t^{\prime}\right)$ for some $p \in\{1, \cdots, n !\}$. By PULSD, it must be $z \ngtr w$ for any $w \in L(v)$. However, we can find $w \in L(v)$ such that $w<z$, as will be proved below.

Let $v(i, j)$ be a vector such that $v_{i}$ and $v_{j}$ are transposed remaining other elements unchanged, i.e., $v(i, j)=\left(v_{1}, \cdots, v_{i-1}, v_{j}, v_{i+1}, \cdots, v_{j-1}, v_{i}, v_{j+1}, \cdots, v_{n}\right)$ for $i, j \in\{1, \cdots, n\}$. We consider

$$
w^{0}=\frac{1}{2} v+\frac{1}{2} v(k, m)
$$




$$
\begin{gathered}
w^{1}=\frac{1}{2} w^{0}+\frac{1}{2} w^{0}(k, m+1) \\
w^{\ell}=\frac{1}{2} w^{\ell-1}+\frac{1}{2} w^{\ell-1}(k, m+\ell), \quad \ell=2, \cdots, n-m .
\end{gathered}
$$

Note that $v_{k} \neq v_{m}, w_{k}^{\ell} \neq w_{m+\ell+1}^{\ell}, \ell=0, \cdots, n-m-1$ by construction. It follows that $w^{0}, w^{1}, \cdots, w^{n-m} \in L(v) \cdot{ }^{27}$ More specifically, $w^{0}, w^{1}$, and $w^{\ell}, \ell=2, \cdots, n-m$ are constructed as follows:

$$
\begin{gathered}
w^{0}: w_{i}^{0}=v_{i} \text { for } i \neq k, m, \\
w_{k}^{0}=w_{m}^{0}=\frac{v_{k}+v_{m}}{2}=\frac{t_{k}^{\uparrow}+\varepsilon t_{m}^{\uparrow}+(1-\varepsilon) e_{k}^{\uparrow}}{2}=\frac{t_{k}^{\uparrow}+e_{k}^{\uparrow}+\varepsilon\left(t_{m}^{\uparrow}-e_{k}^{\uparrow}\right)}{2}
\end{gathered}
$$

(Note that $v_{k}=t_{k}^{\uparrow}<e_{k}^{\uparrow} \leq e_{m}^{\uparrow}<t_{m}^{\uparrow}=v_{m}$ ).

$$
\begin{gathered}
w^{1}: w_{i}^{1}=w_{i}^{0} \text { for } i \neq k, m+1, \\
w_{k}^{1}=w_{m+1}^{1}=\frac{w_{k}^{0}+w_{m+1}^{0}}{2}=\frac{1}{2}\left[\frac{t_{k}^{\uparrow}+e_{k}^{\uparrow}+\varepsilon\left(t_{m}^{\uparrow}-e_{k}^{\uparrow}\right)}{2}+e_{k}^{\uparrow}+\varepsilon\left(t_{m+1}^{\uparrow}-e_{k}^{\uparrow}\right)\right] \\
=\frac{1}{4} t_{k}^{\uparrow}+\frac{3}{4} e_{k}^{\uparrow}+\varepsilon\left[\frac{1}{2}\left(t_{m+1}^{\uparrow}-e_{k}^{\uparrow}\right)+\frac{1}{4}\left(t_{m}^{\uparrow}-e_{k}^{\uparrow}\right)\right] \\
w_{k}^{\ell}=w_{m+\ell}^{\ell}=\frac{w_{k}^{\ell-1}+w_{m+\ell}^{\ell-1}}{2} w_{i}^{\ell}=w_{i}^{\ell-1} \text { for } i \neq k, m+\ell, \quad \ell=2, \cdots, n-m, \\
=\frac{1}{2^{\ell+1}} t_{k}^{\uparrow}+\left(1-\frac{1}{2^{\ell+1}}\right) e_{k}^{\uparrow}+\varepsilon\left[\frac{1}{2}\left(t_{m+\ell}^{\uparrow}-e_{k}^{\uparrow}\right)+\cdots+\frac{1}{2^{\ell+1}}\left(t_{m}^{\uparrow}-e_{k}^{\uparrow}\right)\right]
\end{gathered}
$$

Note that $w_{m}^{n-m}=w_{m}^{0}, w_{m+1}^{n-m}=w_{m+1}^{1}, \cdots, w_{n-1}^{n-m}=w_{n-1}^{n-m-1}$, and, therefore, $w_{m}^{n-m}<w_{m+1}^{n-m}<\cdots<w_{n-1}^{n-m}$. Since $\quad t_{m+\ell}^{\uparrow} \leq t_{n}^{\uparrow} \quad$ or $\quad\left(t_{m+\ell}^{\uparrow}-e_{k}^{\uparrow}\right) \leq\left(t_{n}^{\uparrow}-e_{k}^{\uparrow}\right) \quad$ for $\ell=0, \cdots, n-m$ and $\varepsilon<\frac{1}{2^{n-m+1}-1} \frac{e_{k}^{\uparrow}-t_{k}^{\uparrow}}{t_{n}^{\uparrow}-e_{k}^{\uparrow}}$ by the definition of $\tau$, it follows that

$$
\begin{aligned}
w_{m}^{n-m} & <w_{m+1}^{n-m}<\cdots<w_{n}^{n-m}=w_{k}^{n-m} \\
& =\frac{1}{2^{n-m+1}} t_{k}^{\uparrow}+\left(1-\frac{1}{2^{n-m+1}}\right) e_{k}^{\uparrow}+\varepsilon\left[\frac{1}{2}\left(t_{n}^{\uparrow}-e_{k}^{\uparrow}\right)+\cdots+\frac{1}{2^{n-m+1}}\left(t_{m}^{\uparrow}-e_{k}^{\uparrow}\right)\right] \\
& <\frac{1}{2^{n-m+1}} t_{k}^{\uparrow}+\left(1-\frac{1}{2^{n-m+1}}\right)\left[e_{k}^{\uparrow}+\varepsilon\left(t_{n}^{\uparrow}-e_{k}^{\uparrow}\right)\right]<e_{k}^{\uparrow}=\tau\left(e_{k}^{\uparrow}\right)=z_{k} .
\end{aligned}
$$

Therefore, it is certain that $w_{m+\ell}^{n-m}<z_{m+\ell}$ for $\ell=0, \cdots, n-m$. Combining this observation with the assumption that $w_{i}^{n-m}=v_{i} \leq z_{i}$ for $i \leq m$, we have $w^{n-m}<z$. By construction, $w^{n-m} \in L(v)=L\left(t^{\prime}\right)$ and $z \in S^{\prime}$, which violates PULSD. Q.E.D.

The independence of three axioms characterizing the leximin rule is shown by pointing out that each of the following rules violates one axiom while satisfying the others.

${ }^{27}$ This observation follows straightforwardly from the fact that the product of two bistochastic matrices is also a bistochastic matrix. See Berge $(1963$, Ch.8, $\$ 4)$ on the Theorem 1 (p.181). 
Consider the utilitarian choice rule is the rule $U: \mathbb{C}^{n} \rightarrow \mathbb{R}^{n}$

$$
U(S)=\arg \max _{s \in S} \sum_{i=1}^{n} s_{i}
$$

Certainly, $U(S)$ violates COI.

The leximax rule,

$$
\begin{aligned}
L_{\max }(S)= & \left\{t \in S \mid s \notin S \text { if } \tau_{n}^{\uparrow}<s_{n}^{\uparrow}, \tau_{n}^{\uparrow}=s_{n}^{\uparrow} \text { and } \tau_{n-1}^{\uparrow}<s_{n-1}^{\uparrow}, \cdots,\right. \\
& \text { or } \left.\tau_{2}^{\uparrow}=s_{2}^{\uparrow}, \cdots, \tau_{n}^{\uparrow}=s_{n}^{\uparrow} \text {, and } \tau_{1}^{\uparrow}<s_{1}^{\uparrow}\right\},
\end{aligned}
$$

violates PULSD.

By lemma $L_{\min }(S)$ satisfies GLO, which implies SSO and PULSD. Therefore, it follows from the theorem that GLO together with COI implies and is implied by $L_{\min }(S)$.

Hence, we have the following corollary.

Corollary 1: A choice rule $F(S)$ on $\mathbb{C}^{n}$ satisfies GLO and COI if and only if it is the leximin choice rule $L_{\min }(S)$.

As mentioned above, $L_{\min }(S)$ is not characterized by the set of SSO, LU, and COI in our setting. However, if the feasible set $S$ is restricted to be comprehensive, then LU implies PULSD. Therefore, those three axioms together imply $L_{\min }(S)$, which is easily determined from the proof of Theorem. Hence, we also have the following corollary.

Corollary 2: Given that the feasible set $S$ is comprehensive, a choice rule $F(S)$ on $\mathbb{C}^{n}$ satisfies SSO, LU, and COI if and only if it is the leximin choice rule $L_{\min }(S)$.

\section{Concluding Remarks}

It is interesting to clarify the difference between axiomatizations of the utilitarian and leximin choice rules. Mariotti [8] demonstrated that given a set of feasible social alternatives $A$, which is nonempty and compact, the utilitarian set of $A$ coincides with the baseline independent Suppes-Sen maximal set of $A$ (Theorem 1, p.299). This actually implies that the utilitarian choice rule $U(S)$ is characterized by SSO and the translation invariance (TI) axiom ${ }^{28}$ in our framework.

D'Aspremont and Gevers [4] used social welfare functionals ${ }^{29}$ and characterized the utilitarian and leximin social welfare orderings using the two sets of axioms between which the only difference lies in the invariance axiom. Analogously, in our formulation the utilitarian and leximin choice rules are characterized using the two sets of axioms that are incompatible only in the invariance axiom. Specifically, although both $U(S)$ and $L_{\min }(S)$ satisfy SSO and PULSD, $U(S)$ violates COI and $L_{\min }(S)$ violates TI.

One may ask which axiom, TI or COI, social planner should adopt. Indeed, ${ }^{28} \mathrm{TI}$ is defined as follows: For all $S, S^{\prime} \in \mathbb{C}^{n}$ and for all $\beta \in \mathbb{R}^{n}$ if $S^{\prime}=\left\{s+\beta \in \mathbb{R}^{n} \mid s \in S\right\}$, then $t+\beta \in F\left(S^{\prime}\right)$ whenever $t \in F\left(S^{\prime}\right)$. See Thomson [17].

${ }^{29} \mathrm{As}$ is well-known, the concept of social welfare functional was developed by Sen [13]. 
such an issue is open to discussion.

\section{Acknowledgements}

I appreciate the comments of an anonymous reviewer. Also, I would like to thank Enago for the English language review. In addition, I acknowledge support from Aomori Public University.

\section{Conflicts of Interest}

The authors declare no conflicts of interest regarding the publication of this paper.

\section{References}

[1] Berge, C. (1963) Topological Spaces. MacMillan, New York.

[2] Bossert, W. and Weymark, J.A. (2004) Utility in Social Choice. In: Barbera, S., Hammond, P.J. and Seidl, C., Eds., Handbook of Utility Theory, Vol. 2, Kluwer Academic Publishers, Dordrecht, 1099-1177. https://doi.org/10.1007/978-1-4020-7964-1_7

[3] Dasgupta, P., Sen, A.K. and Starrett, D. (1973) Notes on the Measurement of Inequality. Journal of Economic Theory, 6, 180-187. https://doi.org/10.1016/0022-0531(73)90033-1

[4] D'Aspremont, C. and Gevers, L. (1977) Equity and the Informational Basis of Collective Choice. Review of Economic Studies, 44, 199-209. https://doi.org/10.2307/2297061

[5] Hardy, G., Littlewood, J. and Polya, G. (1934) Inequalities. Cambridge University Press, London.

[6] Madden, P. (1996) Suppes-Sen Dominance, Generalized Lorenz Dominance and the Welfare Economics of Competitive Equilibrium: Some Examples. Journal of Public Economics, 61, 247-262. https://doi.org/10.1016/0047-2727(95)01560-4

[7] Mariotti, M. (1999) Fair Bargains: Distributive Justice and Nash Bargaining Theory. Review of Economic Studies, 66, 733-741. https://doi.org/10.1111/1467-937X.00106

[8] Mariotti, M. (2004) Inequality Aversion, Impartiality and Utilitarianism. Social Choice and Welfare, 17, 45-53. https://doi.org/10.1007/PL00007174

[9] Mori, O. (2017) Characterization of the Lexicographic Egalitarian Solution in the Two-Person Bargaining Problem. Economics Letters, 159, 7-9. https://doi.org/10.1016/j.econlet.2017.06.044

[10] Nielsen, L.T. (1983) Ordinal Interpersonal Comparisons in Bargaining. Econometrica, 51, 219-221. https://doi.org/10.2307/1912255

[11] Ok, E. (1998) Inequality-Averse Collective Choice. Journal of Mathematical Economics, 15, 301-321. https://doi.org/10.1016/S0304-4068(97)00041-4

[12] Saposnik, R. (1983) On Evaluating Income Distributions: Rank-Dominance, the Suppes-Sen Grading Principle of Justice and Pareto Optimality. Public Choice, 40, 329-336. https://doi.org/10.1007/BF00114529

[13] Sen, A.K. (1970) Collective Choice and Social Welfare. North-Holland, Amsterdam.

[14] Sen, A.K. (1973) On Economic Inequality. Clarendon Press, Oxford. https://doi.org/10.1093/0198281935.001.0001

[15] Shorrocks, A. (1983) Ranking Income Distributions. Econometrica, 50, 3-18. 
https://doi.org/10.2307/2554117

[16] Suppes, P. (1966) Some Formal Models of Grading Principles. Syntheses, 16, 284-306. https://doi.org/10.1007/BF00485084

[17] Thomson, W. (1981) Nash's Bargaining Solution and Utilitarian Choice Rules. Econometrica, 49, 535-538. https://doi.org/10.2307/1913329

[18] Thomson, W. (1994) Cooperative Models of Bargaining. In: Aumann, R.J. and Hart, S., Eds., Handbook of Game Theory, Vol. 2, North-Holland, Amsterdam, 1237-1284. https://doi.org/10.1016/S1574-0005(05)80067-0

[19] Von Neumann, J. (1953) A Certain Zero-Sum Two-Person Game Equivalent to the Optimal Assignment Problem. In: Kuhn, H.W. and Tucker, A.W., Eds., Contributions to the Theory of Games, Vol. 2, Princeton University Press, Princeton, 5-12. https://doi.org/10.1515/9781400881970-002 